\title{
Prevalence and Associated Factors of Perinatal Asphyxia in Neonates Admitted to Ayder Comprehensive Specialized Hospital, Northern Ethiopia: A Cross-Sectional Study
}

\author{
Gebrehiwot Teklehaimanot Gebregziabher, ${ }^{1}$ Fikaden Berhe Hadgu $\left(\mathbb{D},{ }^{2}\right.$ \\ and Haftom Temesgen Abebe ${ }^{3}$ \\ ${ }^{1}$ Department of Pediatrics and Child Health, College of Health Sciences, Aksum University, Tigray, Ethiopia \\ ${ }^{2}$ Department of Pediatrics and Child Health, College of Health Sciences, Mekelle University, Tigray, Ethiopia \\ ${ }^{3}$ Department of Biostatistics, School of Public Health, College of Health Sciences, Mekelle University, Tigray, Ethiopia \\ Correspondence should be addressed to Fikaden Berhe Hadgu; fikadenb@gmail.com
}

Received 23 September 2019; Revised 19 November 2019; Accepted 2 December 2019; Published 14 February 2020

Academic Editor: Alessandro Mussa

Copyright (c) 2020 Gebrehiwot Teklehaimanot Gebregziabher et al. This is an open access article distributed under the Creative Commons Attribution License, which permits unrestricted use, distribution, and reproduction in any medium, provided the original work is properly cited.

\begin{abstract}
Background. Perinatal asphyxia is defined as the inability of the newborn to initiate and sustain enough respiration after delivery and is characterized by a marked impairment of gas exchange. It is one of the most common causes of neonatal mortality and morbidity. There are very few studies on perinatal asphyxia in Tigray, and so this study is aimed at assessing the prevalence and associated factors of perinatal asphyxia in Ayder Comprehensive Specialized Hospital NICU, Tigray, Ethiopia. Methods. An institution-based cross-sectional study design was conducted among neonates admitted to Ayder Comprehensive Specialized Hospital from January 1, 2016, to December 30, 2017. Medical records of 267 neonates admitted to the neonatal intensive care unit were selected by a systematic sampling method, and relevant information was collected using a checklist. The data was analyzed using SPSS version 20. Descriptive statistics were computed to determine the prevalence of birth asphyxia and sociodemographic and obstetrics data. Binary logistic regression was used to test associations between the associated factors and perinatal asphyxia. First bivariate analysis was performed to assess the association without controlling the effect of other independent variables. Variables with $P$ value $<0.25$ were fitted to the multivariable binary logistic regression model. Finally, variables with $P$ value $<0.05$ were expressed as associated factors of perinatal asphyxia. Results. Of the 267 neonates, 48 neonates had perinatal asphyxia, giving a prevalence of $18 \%$. Prolonged labor (AOR $=5.19,95 \%$ CI: $1.73-15.63, P=0.003$ ), presence of meconium $(\mathrm{AOR}=4.17,95 \% \mathrm{CI}: 1.34-12.98, P=0.014)$, and preeclampsia $(\mathrm{AOR}=7.94,95 \% \mathrm{CI}: 2.22-28.37$, $P=0.001$ ) were important determinant factors for birth asphyxia. The case fatality rate of perinatal asphyxia was $37.5 \%$. Conclusion and Recommendations. Prevalence and mortality of asphyxia were high. Prolonged labor, presence of meconium, and preeclampsia were determinant factors for birth asphyxia. Early detection and intervention of high-risk mothers should be carried out by health care providers, and mothers should be monitored with partograph during labor.
\end{abstract}

\section{Introduction}

Perinatal asphyxia is defined as the inability of the newborn to initiate and sustain enough respiration after delivery and is characterized by a marked impairment of gas exchange [1].

Perinatal asphyxia is caused by a lack of blood flow or gas exchange to the fetus during late pregnancy, during, or after birth as a neonate. When placental (before birth) or pulmonary (immediate after birth) gas exchange is decreased or stopped altogether, there is partial (hypoxia) or complete (anoxia) lack of oxygen to the vital organs. This causes progressive hypoxemia and hypercapnia. If the hypoxemia is severe enough, the tissues and vital organs (muscle, liver, heart, and ultimately the brain) will develop an oxygen debt. 
Anaerobic glycolysis and lactic acidosis will ensue. Neonatal hypoxic ischemic encephalopathy refers to the neurologic sequelae of perinatal asphyxia $[2,3]$.

The diagnosis of perinatal asphyxia is made when the umbilical cord arterial $\mathrm{pH}$ is $<7$, the APGAR (Appearance, Pulse, Grimace, Activity, and Respiration) score is 0-3 at the fifth minute, and there are central nervous system manifestations like seizures, lethargy, coma, hypotonia, or hypertonia and multisystem organ dysfunction [4].

The incidence of perinatal asphyxia in developed countries is 2 per 1000 live births, but the rate is 10 times greater in developing countries where there is no adequate access to maternal and neonatal care. Of those asphyxiated neonates, $15-20 \%$ will die in the neonatal period and around $25 \%$ of survivors will have permanent neurologic deficits [5].

According to the World Health Organization, perinatal asphyxia is one of the three common causes of under-five child mortality (11\%) following preterm birth (17\%) and pneumonia (15\%) [6]. Africa accounts for $11 \%$ of the world's total population but with more than $25 \%$ of the world's neonatal mortality. Neonatal mortality occurs one in every 4 children in Africa [7]. Because of large neonatal mortalities in Africa, neonatal mortality has remained high globally [810]. Neonates born in sub-Saharan Africa have a substantial risk of perinatal asphyxia. Approximately 280,000 neonatal mortalities occur during the first day of life because of birth asphyxia [7, 8, 11, 12].

In Ethiopia, neonatal mortality accounts for 29/1000 live births. Many of these deaths occur during the first 48 hours of age, and still, the reduction in mortality is low [13]. In Ethiopia, perinatal asphyxia is one of the leading causes of neonatal mortality, accounting for 34\% [14].

Despite this high mortality and morbidity associated with perinatal asphyxia, the prevalence and associated factors of perinatal asphyxia are not well studied in Ethiopia and there is no report on the prevalence and associated factors of perinatal asphyxia in the study area so far. Therefore, this study is aimed at assessing the prevalence and associated factors of perinatal asphyxia among newborns admitted to Ayder Comprehensive Specialized Hospital in Tigray region.

\section{Materials and Methods}

2.1. Study Setting. Ayder Comprehensive Specialized Hospital is found in the Tigray region Mekelle town, which is around $778 \mathrm{~km}$ from the capital city Addis-Ababa. It started as a referral and specialized medical center in 2008 GC. It delivers clinical service to more than a population of 8 million in the catchment areas of Tigray, Afar, and southeastern parts of the Amhara regional state. It provides a broad range of medical services to both in- and outpatient for all age groups. It also serves as a teaching hospital to several medical, dental medicine, nursing, midwifery, public health, pharmacy, anaesthesia, and medical laboratory students in both undergraduate and postgraduate programs. It is the second largest hospital in the nation and has more than 500 inpatient beds in the four major departments (internal medicine, pediatrics, gynaecology and obstetrics, and surgery and other specialties). The pediatrics and child health depart- ment has 18 specialists (general pediatricians) and six subspecialists. There are 43 residents in the department and 30-40 medical interns rotating every three months.

The NICU (neonatal intensive care unit) ward provides service for approximately 200 neonates per month with a total of 43 beds and one room for KMC (kangaroo mother care). There are 65 BSC nurses, 1 neonatologist, 1 general pediatrician, 4 residents, and 8 interns. It is equipped with 4 radiant warmers, 6 incubators, 5 phototherapy devices, and two mechanical ventilation machines.

2.2. Study Design and Period. Institution-based crosssectional study design was used and data collected to include neonates seen from January 1, 2016, to December 30, 2017.

2.2.1. Source Population. The source population was composed of all neonates admitted to Ayder Comprehensive Specialized Hospital.

2.2.2. Study Population. All neonates admitted to Ayder Comprehensive Specialized Hospital, NICU ward, during the study period and who fulfilled the inclusion criteria were included in the study.

2.2.3. Inclusion Criteria. Inclusion criteria include all newborns admitted to Ayder Comprehensive Specialized Hospital with gestational age $\geq 28$ weeks or birth weight $\geq 1000 \mathrm{~g}$.

2.2.4. Exclusion Criteria. Neonates were excluded if they are suffering from major congenital anomalies or syndromes, e.g., NTD (neural tube defect), have incomplete documentation (no maternal or fetal measurement parameters), are kept for observation, and have mothers who took general analgesia.

2.3. Sample Size Determination and Sampling Method. The sample size was determined by using a single proportion formula. The sample size determination formula is

$$
n=\frac{Z^{2} P(1-p)}{d^{2}}
$$

where $n$ is the required sample size, $p$ is the proportion, and $d$ is the level of precision or acceptable error. 95\% confidence interval with a $5 \%$ level of precision and $21.1 \%$ prevalence of perinatal asphyxia were used in a tertiary hospital in Nigeria [15]. The total sample size was calculated to be 256 . With a contingency rate of $10 \%$, the final sample was determined to be 282. Finally, variables with a $P$ value $<0.05$ were expressed as associated factors of perinatal asphyxia. The study subjects were selected by a systematic sampling method, and relevant information was collected using a checklist.

2.4. Study Variables. The dependent and independent variables are provided in Table 1.

2.5. Operational Definitions. Neonates are newborn infants who are less than 28 days.

Perinatal asphyxia is the inability of the newborn to initiate and sustain adequate respiration after delivery. 
TABLE 1: Study variables.

\begin{tabular}{c}
\hline Dependent \\
\hline $\begin{array}{c}\text { Independent } \\
\text { Residence }\end{array}$ \\
Age of neonates at admission \\
Sex of newborn \\
Gestational age in weeks \\
Birth weight in grams \\
Parity \\
Perinatal asphyxia $\quad$ BNC follow-up (antenatal care follow-up) \\
Birth history \\
Type of labor (spontaneous or induced) \\
Duration of labor \\
Place of delivery \\
PROM (premature rupture of membranes) \\
MSAF (meconium-stained amniotic fluid) \\
Fetal presentation \\
Mode of delivery \\
APGAR score (Appearance, Pulse, \\
Drimace, Activity, and Respiration) \\
Duration of hospital stay \\
\hline
\end{tabular}

APGAR score is a score used as a part of early assessment of a newborn.

Perinatal asphyxia is considered when the $5^{\text {th }}$ APGAR score is $<7$ or a neonate did not cry or needed resuscitation.

HIE (hypoxic ischemic encephalopathy) is a central nervous system dysfunction during the neonatal period, and it is due to ischemic and hypoxic insult.

Prolonged labor is the total duration of labor $>24$ hours.

Congenital malformation is a physical defect present in a newborn at birth that results in central nervous system depression.

2.6. Data Collection Procedures. Prestructured data collection format was used to collect the information. Data was collected by medical interns. Relevant information was obtained which includes neonatal information (gender, gestational age, birth weight, and APGAR score), maternal information (age, parity of mothers, residence, place of delivery, mode of delivery, and problems during pregnancy or labor).

2.7. Data Processing and Analysis. Data were entered into SPSS (Statistical Package for the Social Sciences) version 20 (Armonk, NY: IBM Corp), cleaned, coded, and checked for normality and completeness before analysis. Descriptive statistics was used to determine the prevalence of birth asphyxia and sociodemographic as well as obstetrics history. Bivariate and multiple binary logistic regression analysis was carried out to identify the associated factors of PNA. Variables with a $P$ value $<0.25$ during bivariate analysis were included to multivariable logistic regression model. Finally, variables with a $P$ value $<0.05$ were expressed as associated factors for perinatal asphyxia.
2.8. Ethical Considerations. Ethical clearance was obtained from the Institutional Review Board (IRB) of the College of Health Sciences of Mekelle University. Permission was taken from Ayder Comprehensive Specialized Hospital medical director offices; a support letter from the chief clinical director was obtained.

\section{Results}

A total of 3403 neonates were admitted to the NICU during the study period, and a total of 282 neonate medical records were collected using a standardized random sampling approach and 267 (94.7\%) neonate medical records had complete data, and 15 patients were omitted due to incomplete data. Of the 267 neonates, 48 had perinatal asphyxia, giving us a prevalence of $18 \%$. Majority of the study neonates' mothers $(62.2 \%, n=166)$ were aged $20-35$. More than half of the mothers $(57.6 \%, n=154)$ were from urban area. Of the study neonates, $58.8 \%(n=157)$ were males. Most of the neonates $(82.2 \%, n=221)$ were admitted in the first 72 hours of age. The mean age of the study neonates at admission was 2.47 days with SD ( \pm 3 days) and 1.25 ( \pm 0.7 days) for those neonates with PNA. The majority of the mothers of PNA neonates $(28,58.3 \%)$ were between 20 and 35 years of age. Almost all neonates $(98 \%, n=47)$ with PNA were admitted within the first 72 hours of age (Table 1).

3.1. Clinical Characteristics of Study Participants. Majority of the mothers $(60.3 \%, n=161)$ were multiparous. Almost all mothers (98.5\%, $n=263$ ) had ANC follow-up, and 93.6\% $(n=250)$ had normal duration of labor. Of all mothers, 95.9\% $(n=256)$ had intrapartum membrane rupture and $6.4 \%(n=17)$ of them had preeclampsia. With respect to amniotic fluid status, twenty-two (8.2\%) had MSAF. Majority $(61.4 \%, n=164)$ had normal vaginal delivery. Although $5.6 \%(n=15)$ neonates were born with birth weight greater than or equal to $4000 \mathrm{~g}, 59.2 \%(n=158)$ were weighing 2500-3999g.

Among the mothers of neonates with PNA, 52.1\% $(n=25)$ were from rural area, while $58.3 \%(n=28)$ were multiparous. Of all these neonates, $58.3 \%(n=28)$ were born in tertiary hospitals. A significant number of neonates $(8,16.6 \%)$ had MSAF. Most of the mothers $(83.3 \%, n=40)$ had normal labor and delivery history, and 62.5\% $(n=30)$ of them gave birth via SVD. Majority of the asphyxiated neonates $(58.3 \%, n=28)$ were males (Table 2).

3.2. Clinical Features and Outcomes of Asphyxiated Neonates. Thirty-three neonates $(68.75 \%)$ had a score of $4-6$ at the $5^{\text {th }}$ minute, and $27.15 \%(n=13)$ had no record of APGAR score but needed resuscitation (did not cry). More than half (26, $54.17 \%$ ) were admitted with stage II PNA (Table 3). When we see the outcome of asphyxiated neonates, $56.25 \%$ (28) discharged improved, while $37.5 \%(n=18)$ newborns died, resulting in a case fatality rate of $37.5 \%$. From the asphyxiated neonates who died, $61 \%(n=11)$ were stage III, onethird of them $(33.3 \%, n=26)$ were stage II, and one was stage I PNA. The mean hospital stay of PNA patients was 9.42 days with $\mathrm{SD}( \pm 8.81)$. 
TABLE 2: Sociodemographic and clinical characteristics of mothers and neonates at ACSH, Ethiopia, January 1, 2016-December 30, 2017 $(n=267)$.

\begin{tabular}{|c|c|c|c|}
\hline \multirow{2}{*}{ Variables } & \multicolumn{3}{|c|}{ Perinatal asphyxia } \\
\hline & Yes $(48), n(\%)$ & No $(219), n(\%)$ & $n(\%)$ \\
\hline \multicolumn{4}{|l|}{ Age of mothers } \\
\hline$<20$ & $2(4.2)$ & $11(5)$ & $13(4.8)$ \\
\hline $20-35$ & $28(58.3)$ & $138(63)$ & $166(62.2)$ \\
\hline$>35$ & $18(37.5)$ & $70(32)$ & $88(33)$ \\
\hline \multicolumn{4}{|l|}{ Residence of mothers } \\
\hline Urban & $23(47.9)$ & $131(59.8)$ & $154(58.8)$ \\
\hline Rural & $25(52.1)$ & $88(40.1)$ & $113(42.2)$ \\
\hline \multicolumn{4}{|l|}{ Sex of neonates } \\
\hline Male & $28(58.3)$ & $129(58.9)$ & $157(58.8)$ \\
\hline Female & $20(41.7)$ & $90(41.1)$ & $110(41.2)$ \\
\hline \multicolumn{4}{|l|}{ Age of neonate at admission } \\
\hline $0-72 \mathrm{~h}$ & $47(98)$ & $174(79.5)$ & $221(82.2)$ \\
\hline 3-7 days & $1(2)$ & $20(9.1)$ & $21(7.8)$ \\
\hline$>7$ days & $0(0.0)$ & $25(11.4)$ & $25(10)$ \\
\hline \multicolumn{4}{|l|}{ Weight } \\
\hline$\geq 4000$ & $2(4.16)$ & $13(5.9)$ & $15(4.5)$ \\
\hline 2500-3999 & $37(77.1)$ & $121(55.3)$ & $158(59.2)$ \\
\hline $1500-2499$ & $10(20.8)$ & $72(32.9)$ & $82(30.7)$ \\
\hline $1000-1499$ & $2(4.2)$ & $10(4.6)$ & $12(5.6)$ \\
\hline \multicolumn{4}{|l|}{ Parity } \\
\hline Nulliparous & $20(41.7)$ & $86(39.3)$ & $106(39.7)$ \\
\hline Multipara & $28(58.3)$ & $133(60.7)$ & $161(60.3)$ \\
\hline \multicolumn{4}{|l|}{ ANC follow-up } \\
\hline Yes & $48(100)$ & $215(98.2)$ & $263(98.5)$ \\
\hline No & $0(0.0)$ & $4(1.8)$ & $4(1.5)$ \\
\hline \multicolumn{4}{|l|}{ Place of delivery } \\
\hline Tertiary hospital & $28(58.3)$ & $129(58.9)$ & $157(58.8)$ \\
\hline Primary and general hospitals & $6(12.5)$ & $41(18.7)$ & $47(17.6)$ \\
\hline Health center & $13(27.1)$ & $44(16.4)$ & $57(21.3)$ \\
\hline Home delivery & $1(2.1)$ & $5(2.3)$ & $6(2.2)$ \\
\hline \multicolumn{4}{|l|}{ Previous birth history } \\
\hline Abortion & $2(4.2)$ & $18(8.2)$ & $20(7.5)$ \\
\hline Still birth & $1(2.1)$ & $7(3.2)$ & $8(3)$ \\
\hline Neonatal death & $1(2.1)$ & $4(1.8)$ & $5(1.9)$ \\
\hline None & $44(91.7)$ & $190(86.8)$ & $234(87.6)$ \\
\hline \multicolumn{4}{|l|}{ Comorbidity } \\
\hline Preeclampsia & $7(14.6)$ & $10(4.6)$ & $17(6.4)$ \\
\hline $\mathrm{DM}$ & $0(0.0)$ & $6(2.72)$ & $6(2.2)$ \\
\hline None & $41(87.4)$ & $202(92.2)$ & $244(91.4)$ \\
\hline \multicolumn{4}{|l|}{ Type of labor } \\
\hline Spontaneous & $42(87.5)$ & $193(88.1)$ & $235(88)$ \\
\hline Induced & $6(12.5)$ & $24(11.0)$ & $30(11.2)$ \\
\hline Augmented & 0 & $2(0.9)$ & $2(0.8)$ \\
\hline \multicolumn{4}{|l|}{ Duration of labor } \\
\hline Normal & $40(83.3)$ & $210(95.9)$ & $251(94)$ \\
\hline Prolonged & $8(16.6)$ & $9(4.1)$ & $17(6.4)$ \\
\hline
\end{tabular}


TABLE 2: Continued.

\begin{tabular}{|c|c|c|c|}
\hline \multirow{2}{*}{ Variables } & \multicolumn{3}{|c|}{ Perinatal asphyxia } \\
\hline & Yes (48), $n(\%)$ & No (219), $n(\%)$ & $n(\%)$ \\
\hline \multicolumn{4}{|l|}{ PROM } \\
\hline Yes & $1(2.1)$ & $9(4.1)$ & $11(4.1)$ \\
\hline No & $46(97.9)$ & $210(95.9)$ & $256(95.9)$ \\
\hline \multicolumn{4}{|l|}{ MSAF } \\
\hline Yes & $8(16.6)$ & $14(6.4)$ & $22(8.2)$ \\
\hline No & $40(83.3)$ & $205(93.6)$ & $245(91.8)$ \\
\hline \multicolumn{4}{|c|}{ Fetal presentation } \\
\hline Cephalic & $46(95.8)$ & $212(96.8)$ & $258(96.6)$ \\
\hline Noncephalic & $2(4.2)$ & $7(3.2)$ & $9(3.4)$ \\
\hline \multicolumn{4}{|l|}{ Mode of delivery } \\
\hline $\mathrm{C} / \mathrm{S}$ & $16(33.3)$ & $78(35.6)$ & $94(35.2)$ \\
\hline SVD & $30(62.5)$ & $134(61.2)$ & $164(61.4)$ \\
\hline Instrumental & $2(4.2)$ & $7(3.2)$ & $9(3.4)$ \\
\hline \multicolumn{4}{|l|}{ Gestational age } \\
\hline Preterm & $9(18.8)$ & $67(30.6)$ & $76(28.4)$ \\
\hline Term & $38(79.2)$ & $141(64.4)$ & $179(67)$ \\
\hline Postterm & $1(2.1)$ & $11(5.0)$ & $12(4.6)$ \\
\hline
\end{tabular}

Abbreviations: PROM: premature rupture of membrane; MSAF: meconium-stained amniotic fluid; ANC: antenatal care.

TABLE 3: Clinical status of asphyxiated neonates, January 01, 2016December 30, $2017(n=48)$.

\begin{tabular}{lcc}
\hline Clinical factors & Frequency & $\%$ \\
\hline Perinatal asphyxia & & \\
$\quad$ Yes & 48 & 18.0 \\
No & 219 & 82.0 \\
SARNAT stage of PNA & & \\
$\quad$ Stage I & 9 & 18.75 \\
$\quad$ Stage II & 26 & 54.17 \\
Stage III & 13 & 27.1 \\
APGAR score at the $5^{\text {th }}$ minute & & \\
0-3 & 2 & 4.2 \\
4-6 & 33 & 68.75 \\
$\quad$ Needed resuscitation (did not cry) & 13 & 27.1 \\
\hline Abbreviation: PNA: perinatal asphyxia. & &
\end{tabular}

Nine PNA neonates (18.8\%) developed seizure. Of those neonates who had seizure, $66.7 \%(n=6)$ were stage III and $33.3 \%(n=3)$ were stage II.

3.3. Bivariate and Multivariable Analysis. In bivariate regression analysis, residence, gestational age, preeclampsia, place of delivery, birth weight, presence of meconium, and duration of labor were significantly associated factors of PNA (Table 4). But on multivariable logistic regression analysis, preeclampsia, presence of meconium, and duration of labor were statistically significant associated factors of PNA. Neonates born to mothers who had preeclampsia were 7.94 times more likely to develop PNA as compared to neonates from mothers without preeclampsia $(\mathrm{AOR}=7.94,95 \% \mathrm{CI}$ : 2.22-28.37).
Neonates born to mothers with prolonged duration of labor were 5 times more likely to have PNA $(\mathrm{AOR}=5.19$, 95\% CI: 1.73-15.63).

Neonates who were born with meconium-stained amniotic fluid were 4 times more likely to have perinatal asphyxia as compared to those neonates delivered with clear amniotic fluid (AOR = 4.17, 95\% CI: 1.34-12.98).

\section{Discussion}

In the present study, the prevalence of perinatal asphyxia was found to be $18 \%$, which is higher than in developed countries, the latter of which has reduced it to less than $0.1 \%$ [16]. However, similarly high prevalence rate was seen in general hospitals of Tigray, 22.1\% [17]. Moreover, it is comparable with the studies done in other African countries like Gusau, Nigeria (21.1\%) [15], and Dar es Salaam, Tanzania (21.1\%) [18]. But it was lower than the study conducted in Dilla, Southern Ethiopia, which was 32.8\% [19]. This high rate of variation could be due to differences in the methodology, the use of different definitions of birth asphyxia in different settings, the difference in the study facilities, and maybe due to resource disparity or the study participants' economic status. It could also be attributed to the difference in the availability of skilled workers.

In this study, more males than females were affected by perinatal asphyxia (58.3\%). This is consistent with the report from Bangladesh (60.8\%) [20], Dow University of Health Sciences, Karachi (61.3\%) [21], and 60.3\% in Nigeria [22]. This could be explained by the protective effect of the additional " $\mathrm{x}$ " chromosome [23].

The case fatality rate of perinatal asphyxia was $37.5 \%$. This is comparable with the study conducted in Sri Lanka which was $40.6 \%$ [24]. But it is higher than the reports from 
TABLE 4: Bivariate and multivariable logistic regression model showing predictors of birth asphyxia among babies admitted in ACSH, January 1, 2016-December 30, 2017.

\begin{tabular}{|c|c|c|c|c|c|}
\hline Characteristics & PNA & Non-PNA & $\begin{array}{c}\text { At } 95 \% \text { CI } \\
\text { COR }\end{array}$ & $\begin{array}{c}\text { At } 95 \% \mathrm{CI} \\
\text { AOR }\end{array}$ & $P$ value \\
\hline \multicolumn{6}{|l|}{ Preeclampsia } \\
\hline No & 41 & 209 & Ref. & Ref. & \\
\hline Yes & 7 & 10 & $3.57(1.28-9.91)$ & $7.94(2.22-28.37)$ & 0.001 \\
\hline \multicolumn{6}{|l|}{ Residence } \\
\hline Urban & 23 & 131 & Ref. & Ref. & \\
\hline Rural & 25 & 88 & $1.79(1.150-0.98-3.25)$ & $1.7(0.87-3.37)$ & 0.114 \\
\hline Preterm & 9 & 67 & Ref. & Ref. & \\
\hline Term & 38 & 141 & $0.50(0.23-1.09)$ & $0.67(0.22-2.02)$ & 0.486 \\
\hline Postterm & 1 & 11 & $1.48(0.17-12.84)$ & $2.87(0.26-32.47)$ & 0.393 \\
\hline \multicolumn{6}{|l|}{ Place of delivery } \\
\hline Tertiary hospital & 28 & 129 & Ref. & & \\
\hline Primary and general hospitals (district) & 6 & 41 & $1.13(0.46-2.8)$ & $0.84(0.29-2.4)$ & 0.75 \\
\hline Health center & 13 & 44 & $0.61(0.29-1.27)$ & $0.58(0.23-1.47)$ & 0.255 \\
\hline Home & 1 & 5 & $0.99(0.11-8.85)$ & $1.02(0.1-10.34)$ & 0.98 \\
\hline \multicolumn{6}{|l|}{ Birth weight } \\
\hline $2500-399 \mathrm{~g}$ & 34 & 124 & Ref. & Ref. & \\
\hline $1500-2499 \mathrm{~g}$ & 10 & 72 & $1.97(0.92-4.23)$ & $1.59(0.57-4.41)$ & 0.372 \\
\hline $1000-1499 \mathrm{~g}$ & 2 & 10 & $1.37(0.28-6.56)$ & $2.65(0.28-25.01)$ & 0.395 \\
\hline$\geq 4000 \mathrm{~g}$ & 2 & 13 & $1.78(0.38-8.28)$ & $0.56(0.18-5.12)$ & 0.96 \\
\hline \multicolumn{6}{|l|}{ MSAF } \\
\hline Yes & 8 & 14 & $2.92(1.15-7.44)$ & $4.17(1.34-12.98)$ & 0.014 \\
\hline No & 40 & 205 & Ref. & Ref. & \\
\hline \multicolumn{6}{|l|}{ Duration of labor } \\
\hline Normal & 40 & 210 & Ref. & Ref. & \\
\hline Prolonged & 8 & 9 & $4.7(1.7-12.82)$ & $5.19(1.73-15.63)$ & 0.003 \\
\hline
\end{tabular}

Abbreviation: MSAF: meconium-stained amniotic fluid.

Enugu, southeast Nigeria (18\%) [25], Gusau, Nigeria (25.5\%) [15], and Birnin Kudu, Nigeria (10.3\%) [26]. However, the frequency found in this study is lower than the $62.5 \%$ fatality rate from Dar es Salaam, Tanzania [18].

The disparity could be attributable to the difference in hospital setup (some may be better equipped) and the difference in health care provider skills and birth asphyxia severity. Although this study has reported high fatality rate, perinatal asphyxia has contributed to a low overall neonatal mortality rate $(6.7 \%, N=18)$ which is lower than what has been reported in other studies.

Most of the neonates with stage III perinatal asphyxia $(84.6 \%)$ died. This is higher than the reported $66.7 \%$ from Enugu, southeast Nigeria [25], and $44.4 \%$ from Karnataka, India [27]. The reason could be due to the difference in the health facility setup and the presence of multiorgan failure associated with hypoxic-ischemic encephalopathy.

The current study revealed that the odds of developing perinatal asphyxia was 4 times higher in neonates of mothers who had meconium-stained amniotic fluid than those without meconium-stained amniotic fluid. Such was demonstrated in the general hospitals of Tigray [17] and also $[20,28,29]$. MASF is often associated with fetal hypoxia which promotes meconium discharge in amniotic fluid, gasping and aspiration of MSAF, and also changes in the vascular muscular of pulmonary blood vessels of the fetus [30].

The odds of developing perinatal asphyxia was 5.19 times higher in a newborn whose mother had prolonged duration of labor. This is consistent with reports from different hospitals in Ethiopia including general hospitals in Tigray, Dessie, and Dire Dawa $[17,31,32]$ and in other African countries like Cameroon [33] and Bayero University Kano, Nigeria [22]. The reason could be delayed labor which might be causing the fetus to be involved in labor for a long time that carries a higher risk of birth trauma and asphyxia [34]. Strict follow-up of laboring mother with partograph may ameliorate the situation.

Neonates born to mothers who had preeclampsia were 7.94 times more likely to have perinatal asphyxia than neonates from mothers without preeclampsia. This is in agreement with studies done in universities in Nigeria [22], Nairobi [35], and Pakistan [36]. The reason why preeclampsia is a risk factor for perinatal asphyxia could be due to decreased blood flow to the fetus that may lead to hypoxia and finally perinatal asphyxia. 
As this study has shown high prevalence and also alarmingly significant mortality in neonates with PNA, good obstetric interventions and proper care of neonates in the neonatal intensive care unit are mandatory.

4.1. Strength of the Study. This is the first PNA study in our hospital, and it was able to show the prevalence, associated factors, and outcome of asphyxiated neonates. The study was designed with random sampling technique. Moreover, neonates were included from both rural and urban areas of residence.

4.2. Limitations of the Study. This study had some important limitations because it was conducted in a tertiary care hospital where significant numbers of patients were referred being critical; therefore, this prevalence may not reflect the overall prevalence of the community.

Furthermore, this study does not show cause-and-effect relationships because of the cross-sectional study design.

Measurement of fetal or neonatal arterial blood gas would give a stricter and more precise definition of PNA but our hospital's setup could not give such services because of resource constraints.

\section{Conclusion}

In conclusion, the prevalence of perinatal asphyxia was high. The case fatality rate of perinatal asphyxia was alarmingly high. Prolonged labor, presence of meconium-stained amniotic fluid, and preeclampsia were predictors of perinatal asphyxia. Early detection and intervention of highrisk mothers should be carried out by health care providers, and mothers should be monitored with partograph during labor.

\section{Data Availability}

All important data are included in the manuscript.

\section{Conflicts of Interest}

The authors declare that there is no conflict of interest regarding the publication of this paper.

\section{Acknowledgments}

We are grateful to Dr. Abraha Gebreegziabher who helped us in proofreading and editing the manuscript.

\section{References}

[1] ACOG, Neonatal Encephalopathy and Neurologic Outcome, American Academy of Pediatrics, Washington, DC, 2nd edition, 2014.

[2] M. Sugiura-Ogasawara, T. Ebara, Y. Yamada et al., "Adverse pregnancy and perinatal outcome in patients with recurrent pregnancy loss: multiple imputation analyses with propensity score adjustment applied to a large-scale birth cohort of the Japan Environment and Children's Study," American Journal of Reproductive Immunology, vol. 81, no. 1, article e13072, 2019.
[3] M. Hakobyan, K. P. Dijkman, S. Laroche et al., "Outcome of infants with therapeutic hypothermia after perinatal asphyxia and Early-Onset sepsis," Neonatology, vol. 115, no. 2, pp. 127-133, 2019.

[4] B. Azra Haider and Z. A. Bhutta, "Birth asphyxia in developing countries: current status and public health implications," Current Problems in Pediatric and Adolescent Health Care, vol. 36, no. 5, pp. 178-188, 2006.

[5] D. Odd, A. Heep, K. Luyt, and T. Draycott, "Hypoxic-ischemic brain injury: planned delivery before intrapartum events," Journal of Neonatal-Perinatal Medicine, vol. 10, no. 4, pp. 347-353, 2017.

[6] WHO, "Global health observatory data repository world health organization," 2015, August 2019, http://apps.who.int/ ghodata/.

[7] J. Lawn, P. Mongi, and S. Cousens, "Africa's newborns-counting them and making them count," Opportunities for Africa's Newborns, 2011.

[8] J. E. Lawn, S. Cousens, and J. Zupan, "4 million neonatal deaths: when? Where? Why?,” The Lancet, vol. 365, no. 9462, pp. 891-900, 2005.

[9] J. E. Lawn, H. Blencowe, S. Oza et al., "Every newborn: progress, priorities, and potential beyond survival," The Lancet, vol. 384, no. 9938, pp. 189-205, 2014.

[10] J. E. Lawn, A. Manandar, R. A. Haws, and G. L. Darmstadt, "Reducing one million child deaths from birth asphyxia - a survey of health systems gaps and priorities," Health Research Policy and Systems, vol. 5, no. 1, article 4, 2007.

[11] J. Wu, Levels \& Trends in Child Mortality, UNICEF, WHO, World Bank, United Nations, Department of Economic and Social Affairs, 2013.

[12] UNICEF, Committing to Child Survival: A Promise Renewed, 2013, https://www.unicef.org/publications/files/2013_APR_ Progress_Report_Summary_EN_10_Sept_2013.pdf.

[13] Central Statistical Agency (CSA) and ICF, Ethiopia Demographic and Health Survey 2016, CSA and ICF, Addis Ababa, Ethiopia. Rockville, Maryland, USA, 2016.

[14] United Nations Children's Fund, World Health Organization, World Bank and United Nations, Levels \& trends in child mortality report, New York (NY), Geneva and Washington (DC), 2015, http://www.unicef.org/publications/?esSept_15.pdf.

[15] B. G. Ilah, M. S. Aminu, A. Musa, M. B. Adelakun, A. O. Adeniji, and T. Kolawole, "Prevalence and risk factors for perinatal asphyxia as seen at a specialist hospital in Gusau, Nigeria," Sub-Saharan African Journal of Medicine, vol. 2, no. 2, p. 64, 2015.

[16] Saving Newborn Lives, The state of the world's newborn: a report from Saving Newborn Lives, SAVE the Children, Washington, D.C., 2001.

[17] G. Gebreheat, T. Tsegay, D. Kiros et al., "Prevalence and associated factors of perinatal asphyxia among neonates in general hospitals of Tigray, Ethiopia, 2018," BioMed Research International, vol. 2018, Article ID 5351010, 7 pages, 2018.

[18] A. Sepeku and T. W. Kohi, "Treatment outcomes of neonatal asphyxia at a national hospital in Dar es Salaam, Tanzania," Africa Journal of Nursing and Midwifery, vol. 13, no. 2, pp. 43-56, 2011.

[19] A. Alemu, G. Melaku, G. B. Abera, and A. Damte, "Prevalence and associated factors of perinatal asphyxia among newborns in Dilla University referral hospital, Southern 
Ethiopia- 2017," Pediatric Health, Medicine and Therapeutics, vol. Volume 10, pp. 69-74, 2019.

[20] M. Solayman, S. Hoque, T. Akber, M. I. Islam, and M. A. Islam, "Prevalence of perinatal asphyxia with evaluation of associated risk factors in a rural tertiary level hospital," KYAMC Journal, vol. 8, no. 1, pp. 43-48, 2017.

[21] H. M. Aslam, S. Saleem, R. Afzal et al., "Risk factors of birth asphyxia," Italian Journal of Pediatrics, vol. 40, no. 1, p. 94, 2014.

[22] I. Aliyu, T. O. Lawal, and B. Onankpa, "Prevalence and outcome of perinatal asphyxia: our experience in a semi-urban setting," Tropical Journal of Medical Research, vol. 20, no. 2, pp. 161-165, 2017.

[23] R. Pongou, "Why is infant mortality higher in boys than in girls? A new hypothesis based on preconception environment and evidence from a large sample of twins," Demography, vol. 50, no. 2, pp. 421-444, 2013.

[24] R. M. Meshram and C. M. Bokade, "Risk factors for mortality in birth asphyxia of outborn neonates: a prospective observational study," Sri Lanka Journal of Child Health, vol. 48, no. 1, pp. 26-32, 2019.

[25] E. Uchenna, N. I. Asinobi, C. D. I. Osuorah et al., "Incidence and predictors of mortality among newborns with perinatal asphyxia: a 4-year prospective study of newborns delivered in health care facilities in Enugu, South-East Nigeria," Clinical Medicine Insights: Pediatrics, vol. 11, article $117955651774664,2017$.

[26] U. A. Idris, "Perinatal asphyxia in term neonates seen in Federal Medical Centre, Birnin Kudu, Nigeria," International Journal of Health \& Allied Sciences, vol. 8, no. 1, pp. 44-47, 2019.

[27] R. S. Pattar, A. Raj, and B. C. Yelamali, "Incidence of multiorgan dysfunction in perinatal asphyxia," International Journal of Contemporary Pediatrics, vol. 2, pp. 428-432, 2015.

[28] C. Pitsawong and P. Panichkul, "Risk factors associated with birth asphyxia in Phramongkutklao Hospital," Thai Journal of Obstetrics and Gynaecology, vol. 19, no. 4, pp. 165-171, 2011.

[29] M. Krishnan and S. Padarthi, "A prospective study on intrapartum risk factors for birth asphyxia," IOSR Journal of Dental and Medical Sciences, vol. 15, no. 9, pp. 04-07, 2016.

[30] J. M. Whitfield, D. S. Charsha, and A. Chiruvolu, "Prevention of meconium aspiration syndrome: an update and the Baylor experience," Baylor University Medical Center Proceedings, vol. 22, pp. 128-131, 2009.

[31] Y. Kibret, G. Hailu, and K. Angaw, "Determinants of birthasphyxia among newborns in Dessie Town Hospitals, North Central Ethiopia," International journal of Sexual Health and Reproductive Health, vol. 1, no. 1, pp. 1-12, 2018.

[32] N. A. Ibrahim, A. Muhye, and S. Abdulie, "Prevalence of birth asphyxia and associated factors among neonates delivered in Dilchora Referral Hospital, in Dire Dawa, Eastern Ethiopia," Clinics Mother Child Health, vol. 14, no. 4, p. 279, 2017.

[33] A. Chiabi, S. Nguefack, M. A. Evelyne et al., "Risk factors for birth asphyxia in an urban health facility in Cameroon," Iranian journal of child neurology, vol. 7, no. 3, pp. 46-54, 2013.

[34] Reiter \& Walsh, PC, Birth Asphyxia and HIE (Hypoxic-Ischemic Encephalopathy) from Prolonged Labor, American Baby and Child Law Centers, Bloomfield Hills, MI, USA, 2010, https:// www.abclawcenters.com/practice-areas/prenatal-birth-injuries/ fetus-or-newborn-medical-problems/birth-asphyxia-lawyers/ birth-asphyxia-hypoxic-ischemic-encephalopathy-hie-oftencaused-prolonged-labor/.

[35] E. K. Kibai, Perinatal factors associated with birth asphyxia among neonates in maternity ward Kakamega County Reera O Hospital, Kenya, MMUST Digital Repository, 2017.

[36] F. Tabassum, A. Rizvi, S. Ariff, S. Soofi, and Z. A. Bhutta, "Risk factors associated with birth asphyxia in rural district Matiari, Pakistan: a case control study," International Journal of Clinical Medicine, vol. 5, no. 21, pp. 1430-1441, 2014. 